\title{
Effects of material hardship on depression among adults in South Korea: insights from by the Korea Welfare Panel Study 2008- 2017
}

Soo Hyun Kang ${ }^{1,2}$, Selin Kim ${ }^{1,2}$, Eun-Cheol Park ${ }^{2,3}$ and Sung-In Jang ${ }^{2,3^{*}}$

\begin{abstract}
Background: Low socioeconomic status deemed by income-based measures is a risk factor for depression. Material hardship is commonly used as a multidimensional socioeconomic indicator to identify the struggles that low-income households encounter that are not captured by conventional income-based measures. The aim of this study was to examine the effect of material hardship on depression.

Methods: We used wave 3 (2008) to wave 12 (2017) panel data collected by the Korea Welfare Panel Study. The material hardship measure included six dimensions: food, housing, medical care, paying utility bills, education, and financial hardship. Depression was measured using the Center for Epidemiologic Studies Depression Scale (CESD-11). A generalised estimating equation model was applied to test the causal association between material hardship and log transferred CESD-11.

Results: The first time point comprised 3,866 participants. Those who continually experienced material hardship had higher depression scores (male: $\beta=2.82$, female: $\beta=3.98, p$-value: $<.0001$ ). Food hardship was the most critical risk factor (male: $\beta=3.29$, female: $\beta=4.05$, $p$-value: $<.0001$ ).

Conclusions: Material hardship is associated with increased risk of depression, especially food hardship. We should consider guaranteeing food security, and community and policy makers should consider material hardship in their approach when identifying low-income populations at high risk for depression.
\end{abstract}

Keywords: Depression, Socioeconomic status, Material Hardship

\section{Background}

South Korea has witnessed a dramatic increase in income inequality and poverty in the past two decades, with the Gini coefficient skyrocketing from 0.28 to 0.35 within this short period of time [1]. As the proportion of females and irregular workers (contractors) in labour market have become higher, their lower income compared to males

\footnotetext{
*Correspondence: JANGSI@yuhs.ac

${ }^{2}$ Institute of Health Services Research, Yonsei University, Seoul, Republic of Korea

Full list of author information is available at the end of the article
}

and regular workers are related to income inequality [2]. This inequality has led to concerns regarding poverty and health inequality in low-income households. Aging society is another cause [2]. Korean government has poverty reducing systems, such as the National Basic Livelihood Guarantee System (NBLGS). However, the proportion of beneficiaries is generally only $3 \%$ in population because of the strict criteria regarding income level and absence of supportable family members. The rate of beneficiaries in population whose income level met the criteria was only $52.61 \%$ which is assumed as the huge blind spot of original author(s) and the source, provide a link to the Creative Commons licence, and indicate if changes were made. The images or other third party material in this article are included in the article's Creative Commons licence, unless indicated otherwise in a credit line to the material. If material is not included in the article's Creative Commons licence and your intended use is not permitted by statutory regulation or exceeds the permitted use, you will need to obtain permission directly from the copyright holder. To view a copy of this licence, visit http://creativecommons.org/licenses/by/4.0/. The Creative Commons Public Domain Dedication waiver (http://creativeco mmons.org/publicdomain/zero/1.0/) applies to the data made available in this article, unless otherwise stated in a credit line to the data. 
the system [3]. Hence, the public social spending of GDP in Korea was only $12.2 \%$ in 2019 , while the average of Organisation for Economic Co-operation and Development (OECD) was 20.0\% [4].

Socioeconomic status, which is typically measured by income, has the advantage of providing an objective measure and definition of low-income households. However, income can overlook the daily struggles that these households may experience and only indirectly estimate what low income actually means for people [5, 6]. As numerous studies have highlighted various limitations of the one-dimensional and conventional simplistic income-based measures of socioeconomic status, material hardship $(\mathrm{MH})$ was introduced to capture the multidimensional aspect of the financial challenges people encounter daily [7].

MH was developed by Sen $[8]$ and Townsend $[9,10]$ to measure the living conditions of the poor. They argued that different groups of people and households have different consumption habits and face different demands. Beverly [11] defined $\mathrm{MH}$ as the failure to be able to consume minimal levels of basic services and goods such as food, housing, basic goods, clothing, and medical care mainly due to financial challenges. Researchers have found that there was little overlap between the groups of people deemed poor by the income-based measure and those deemed poor by the MH measure [12]. Thus, $\mathrm{MH}$ is used as a socioeconomic indicator to identify the struggles that low-income households actually encounter, unlike the conventional income-based measure.

The World Health Organization issued a report on mental health which stated that 'No group is immune to mental disorders, but the risk is higher among the poor, homeless, the unemployed, and persons with low education' [13]. Heflin and Iceland [14] reported a strong relationship between $\mathrm{MH}$, especially when encountering problems with paying bills and having one's phone disconnected, and depression in the U.S. data. In Korea, numerous researchers have reported the association between low socioeconomic status and mental disorders, such as depressive symptoms $[15,16]$ and suicidal ideation [17] and attempts [18]. However, in Korea, onedimensional and conventional income-based measures of socioeconomic status have been much more commonly used than multidimensional measures in studies examining the association between mental disorders and low socioeconomic status.

Although, previous studies have shown the relationship between poverty and depression, in Korea, their relationship to $\mathrm{MH}$, which is an extended concept of poverty, and depression has not been thoroughly assessed. Therefore, the aim of this study was to examine the effect of $\mathrm{MH}$ on depression.

\section{Methods}

\section{Data and population}

We used data from the Korea Welfare Panel Study (KoWePS) conducted by the Korea Institute for Health and Social Affairs and Seoul National University. The participants were recruited using two-stage stratified cluster sampling in 2006. These data are considered suitable for consideration in low-income policies or poverty studies because approximately $50 \%$ of the sample were low-income earners with a median income of $60 \%$ or less. The present sample was drawn from waves 3 (2008) to 12 (2017) of the study. The 2009 baseline data included 16,255 individuals from 6,207 households.

To analyse the effect of $\mathrm{MH}$, we excluded participants under 19 years of age (3,679 excluded) and individuals with missing data $(8,130$ excluded). In addition, 580 individuals who did not respond to the $\mathrm{MH}$ questions in the prior year (2008) were excluded. Thus, the final 2009 data included a total of 3,866 individuals: 3,568 males and 298 females.

\section{Measures}

The primary outcome was depression, which was measured based on the 11-item version of the Center for Epidemiologic Studies Depression Scale (CESD-11). The CESD-11 is a shorter version of the original 20-item version and is a self-reported screening tool which is well validated [19]. In KoWePS, respondents reported symptoms they had experienced during the previous week using a version of the scale that had been translated into Korean and validated by Cho and Kim [20]. The scale used a four-point Likert scale that ranged from 0 to 3 , and the total score was calculated by adding up all responses. Higher scores indicated greater distress; a score above 16 is usually considered indicative of depression [21].

The MH was the key independent variable. In KoWePS, the participants answered 13 questions on whether their household was affected by $\mathrm{MH}$ in the prior year. The questions covered the following areas: (1) ran out of food and could not afford to buy more, (2) could not afford balanced meals, (3) adults in the household skipped meals or did not have enough to eat, (4) ate less than needed because could not afford to buy enough food, (5) were hungry but did not eat because could not afford to buy food to eat, (6) had electricity, telephone, or water disconnected because of unpaid bills, (7) unable to pay utility bills before the due date, (8) unable to pay rent for over two months or had to move out because of unpaid rent or inability to pay rent, (9) unable to sufficiently heat the home during the winter, (10) myself or other family members needed to see a doctor but could not afford to go, (11) unable to pay the national health insurance premium and lost eligibility for national health 
insurance, (12) had problems with credit, and (13) unable to pay children's public education tuition. All items were binary variables (Yes $=1 ; \mathrm{No}=2$ ) except two of the foodrelated hardship items (questions 1 and 2), which had three responses (i.e. 'often', 'sometimes', and 'never'). We coded them as binary indicators by combining the 'sometimes' and 'often' responses into one category. The 13 $\mathrm{MH}$ items capture the four dimensions of $\mathrm{MH}$ (i.e. food hardship, difficulty paying bills, lack of medical care, and unstable housing) $[7,14]$, which have been widely used in research. The unique aspects of MH in the South Korean context, such as having problems with credit and the inability to pay children's tuition, were also included.

If participants answered 'Yes' to at least one of the 13 questions, we considered they had experienced MH. A change in $\mathrm{MH}$ over one year was classified into four categories: (1) 'No $\rightarrow$ No' for not having experienced $\mathrm{MH}$ for the whole study period; (2) 'No $\rightarrow$ Yes' for not having experienced $\mathrm{MH}$ in the prior year but having experienced $\mathrm{MH}$ in the following year; (3) 'Yes $\rightarrow$ No' for having experienced $\mathrm{MH}$ in the prior year and not having experienced $\mathrm{MH}$ in the following year; (4) 'Yes $\rightarrow$ Yes' for having experienced $\mathrm{MH}$ in both the prior year and following years of the study.

Demographic, socioeconomic, and health related factors were included. Demographic variables included gender, age, and region. Socioeconomic variables included education level, marital status, NBLGS beneficiary status, and income level (quartile). Health related factors included smoking, alcohol intake, disability status, prior year depression status, and chronic diseases.

\section{Statistical analysis}

We calculated the distribution of the participants' general characteristics at baseline. Analysis of variance (ANOVA) was used to assess the differences in mean CESD-11 scores among the 3,866 sampled subjects at the first time point $(2008 \rightarrow 2009)$. To analyse the effect of $\mathrm{MH}$ on depression using the repeated measured panel data, we used the generalised estimating equation (GEE) model with exchangeable correlation matrix. The GEE model is more efficient and provides unbiased regression estimates for use in analysing repeated measures research designs with non-normal response variables [22]. The GEE model considers the correlation within the subjects to yield the regression coefficient ( $\beta$ ), the standard error of the coefficient (SE), and the corresponding $p$-value by doing We evaluated whether the MH transition in each prior year was associated with their depression in each subsequent year over two consecutive years from 2008 and 2009 till 2016 and 2017. Thus, total nine time points were used in this study. Statistical analyses were performed using the GENMOD procedure with 'repeated subject' option in SAS, version 9.4 (SAS Institute, Inc., Cary, NC, USA). The results were considered statically significant if the $p$-value was less than 0.05 .

\section{Results}

Table 1 presents participants' general characteristics at the first change time point $(2008 \rightarrow 2009)$. Of the 3,866 participants, $71.2 \%$ of the male and $47.7 \%$ of the female participants were in the No $\rightarrow$ No group. The No $\rightarrow$ Yes group was the smallest, accounting for $6.8 \%$ of the male and $6.7 \%$ of the female participants. Male participants in the Yes $\rightarrow$ Yes group showed the highest mean score on the CESD-11 (No $\rightarrow$ No: 6.79; No $\rightarrow$ Yes: 10.86; Yes $\rightarrow$ No: 9.56; Yes $\rightarrow$ Yes: 12.77). Female participants in the No $\rightarrow$ Yes group showed the highest mean scores on the CESD-11 (No $\rightarrow$ No: 14.14; No $\rightarrow$ Yes: 19.18; Yes $\rightarrow$ No: 15.27; Yes $\rightarrow$ Yes: 17.95). Generally, female participants demonstrated higher mean scores on the CESD-11 than males.

Table 2 shows the results of the GEE model analysis of factors associated with depression. The $\mathrm{MH}$ Yes $\rightarrow$ Yes group had higher depression scores (male: $\beta=2.82$, female: $\beta=3.98$ ) than the No $\rightarrow$ No group. Moreover, the $\mathrm{MH}$ Yes $\rightarrow$ No group had the higher depression scores as the second (male: $\beta=2.28$, female: $\beta=3.41$ ), while the $\mathrm{MH} \mathrm{No} \rightarrow$ Yes group had only slightly higher depression scores (male: $\beta=0.45$, female: $\beta=0.84$ ) than the No $\rightarrow$ No group. Depression scores were higher among participants who experienced depression (having a CESD-11 score of minimum 16) in the prior year (male: $\beta=3.14$, female: $\beta=2.93$ ) than among those who did not experience depression in the prior year. Participants with a lower income level tended to have higher depression scores (low: male, $\beta=1.98$, female, $\beta=2.05$; lower middle: male, $\beta=1.00$, female, $\beta=1.13$; upper middle: male, $\beta=0.56$, female, $\beta=0.53$ ) than those with a higher income level. Depression scores were higher among the disabled participants (male: $\beta=0.96$, female: $\beta=1.09$ ), especially the severely disabled (male: $\beta=2.62$, female: $\beta=2.05$ ), compared to the non-disabled participants. Further, unmarried participants, NBLGS beneficiaries, current smokers, and participates with chronic diseases tended to have higher CESD-11 scores. Only male participants who consumed alcohol less than 4 times a week (once a week or less: $\beta=-0.59 ; 2-3$ times a week: $\beta=-0.70$ ) had lower CESD-11 scores than non-drinkers.

Table 3 outlines the subgroup analysis regarding the effects of $\mathrm{MH}$ on depression stratified by socioeconomic and health-related variables. Generally, the $\mathrm{MH}$ Yes $\rightarrow$ Yes group had the highest depression status risk, followed by the No $\rightarrow$ Yes group. Although some were not statistically significant, the Yes $\rightarrow$ No groups also had a slightly higher risk of depression than the $\mathrm{No} \rightarrow \mathrm{No}$ 
Table 1 Participants' general characteristics at the first change time point (2008 $\rightarrow$ 2009)

\begin{tabular}{|c|c|c|c|c|c|c|c|c|c|c|c|c|}
\hline \multirow[t]{3}{*}{ Variables } & \multicolumn{12}{|c|}{ CESD-11 } \\
\hline & \multicolumn{5}{|l|}{ Male } & \multirow[t]{2}{*}{$P$ value } & \multicolumn{5}{|c|}{ Female } & \multirow[t]{2}{*}{$P$ value } \\
\hline & $\mathrm{N}$ & $\%$ & MEANS & \pm & SD & & $\mathrm{N}$ & $\%$ & MEANS & \pm & SD & \\
\hline TOTAL & 3,568 & 100 & 8.02 & \pm & 9.06 & & 298 & 100 & 15.77 & \pm & 12.57 & \\
\hline \multicolumn{13}{|c|}{ Change in material hardship $(2008 \rightarrow 2009)$} \\
\hline $\mathrm{No} \rightarrow \mathrm{No}$ & 2,539 & 71.2 & 6.79 & \pm & 8.05 & $<.0001$ & 142 & 47.7 & 14.14 & \pm & 12.29 & 0.3803 \\
\hline $\mathrm{No} \rightarrow \mathrm{Yes}$ & 243 & 6.8 & 10.86 & \pm & 10.32 & & 20 & 6.7 & 19.18 & \pm & 14.29 & \\
\hline Yes $\rightarrow$ No & 403 & 11.3 & 9.56 & \pm & 9.96 & & 50 & 16.8 & 15.27 & \pm & 12.12 & \\
\hline Yes $\rightarrow$ Yes & 383 & 10.7 & 12.77 & \pm & 11.08 & & 86 & 28.9 & 17.95 & \pm & 12.63 & \\
\hline \multicolumn{13}{|l|}{ Prior year depression status (2008) } \\
\hline No & 2,924 & 82.0 & 6.31 & \pm & 7.34 & $<.0001$ & 164 & 55.0 & 10.51 & \pm & 10.47 & $<.0001$ \\
\hline Yes & 644 & 18.0 & 15.79 & \pm & 11.67 & & 134 & 45.0 & 22.20 & \pm & 11.96 & \\
\hline \multicolumn{13}{|l|}{ Age (years) } \\
\hline $19-29$ & 280 & 7.8 & 7.03 & \pm & 8.88 & 0.1147 & 22 & 7.4 & 17.44 & \pm & 14.97 & 0.0156 \\
\hline $30-39$ & 740 & 20.7 & 6.05 & \pm & 7.18 & & 21 & 7.0 & 9.61 & \pm & 10.65 & \\
\hline $40-49$ & 752 & 21.1 & 7.55 & \pm & 9.02 & & 34 & 11.4 & 12.78 & \pm & 10.82 & \\
\hline $50-59$ & 535 & 15.0 & 8.44 & \pm & 9.74 & & 40 & 13.4 & 14.18 & \pm & 12.82 & \\
\hline $60-69$ & 624 & 17.5 & 8.73 & \pm & 9.51 & & 48 & 16.1 & 21.63 & \pm & 13.38 & \\
\hline$\geq 70$ & 637 & 17.9 & 10.26 & \pm & 9.49 & & 133 & 44.6 & 15.58 & \pm & 11.80 & \\
\hline \multicolumn{13}{|l|}{ Region } \\
\hline Metropolitan & 1,508 & 42.3 & 8.14 & \pm & 9.35 & 0.0617 & 126 & 42.3 & 16.46 & \pm & 13.63 & 0.2671 \\
\hline Rural & 2,060 & 57.7 & 7.93 & \pm & 8.84 & & 172 & 57.7 & 15.25 & \pm & 11.75 & \\
\hline \multicolumn{13}{|l|}{ Education level } \\
\hline Middle school or under & 1,299 & 36.4 & 9.99 & \pm & 9.85 & 0.1855 & 225 & 75.5 & 16.47 & \pm & 12.34 & 0.0614 \\
\hline High school & 1,280 & 35.9 & 7.63 & \pm & 8.79 & & 44 & 14.8 & 14.79 & \pm & 13.42 & \\
\hline College or above & 989 & 27.7 & 5.93 & \pm & 7.68 & & 29 & 9.7 & 11.79 & \pm & 12.67 & \\
\hline \multicolumn{13}{|l|}{ Marital status } \\
\hline Living w spouse & 2,726 & 76.4 & 7.42 & \pm & 8.36 & $<.0001$ & 73 & 24.5 & 13.60 & \pm & 12.55 & 0.4768 \\
\hline Living w/o spouse & 842 & 23.6 & 9.96 & \pm & 10.78 & & 225 & 75.5 & 16.47 & \pm & 12.53 & \\
\hline \multicolumn{13}{|l|}{ Income level } \\
\hline Low & 840 & 23.5 & 10.95 & \pm & 10.25 & 0.0195 & 111 & 37.2 & 18.85 & \pm & 12.72 & 0.1082 \\
\hline Lower middle & 867 & 24.3 & 8.65 & \pm & 9.42 & & 85 & 28.5 & 16.49 & \pm & 12.15 & \\
\hline Upper middle & 883 & 24.7 & 7.17 & \pm & 8.17 & & 63 & 21.1 & 12.78 & \pm & 11.57 & \\
\hline High & 978 & 27.4 & 5.71 & \pm & 7.51 & & 39 & 13.1 & 10.21 & \pm & 12.13 & \\
\hline \multicolumn{13}{|l|}{ NBLGS* status } \\
\hline Non-beneficiary & 3,255 & 91.2 & 7.38 & \pm & 8.39 & 0.0003 & 217 & 72.8 & 14.38 & \pm & 12.06 & 0.0976 \\
\hline Beneficiary & 313 & 8.8 & 14.73 & \pm & 12.42 & & 81 & 27.2 & 19.48 & \pm & 13.22 & \\
\hline \multicolumn{13}{|l|}{ Current smoking status } \\
\hline Non-smoker & 2,159 & 60.5 & 8.32 & \pm & 9.18 & $<.0001$ & 195 & 65.4 & 15.79 & \pm & 12.65 & 0.4713 \\
\hline Current smoker & 1,409 & 39.5 & 7.57 & \pm & 8.84 & & 103 & 34.6 & 15.73 & \pm & 12.49 & \\
\hline \multicolumn{13}{|l|}{ Alcohol consumption } \\
\hline Non-drinkers & 860 & 24.1 & 10.35 & \pm & 10.34 & 0.0013 & 167 & 56.0 & 17.00 & \pm & 12.08 & 0.1284 \\
\hline Once a week or less & 1,260 & 35.3 & 6.97 & \pm & 8.34 & & 93 & 31.2 & 11.98 & \pm & 11.77 & \\
\hline 2-3 times a week & 825 & 23.1 & 7.08 & \pm & 8.46 & & 21 & 7.0 & 20.00 & \pm & 13.80 & \\
\hline More than 4 times a week & 623 & 17.5 & 8.19 & \pm & 8.73 & & 17 & 5.7 & 19.14 & \pm & 15.85 & \\
\hline \multicolumn{13}{|l|}{ Disability status } \\
\hline Not disabled & 3,117 & 87.4 & 7.45 & \pm & 8.59 & 0.0001 & 269 & 90.3 & 15.57 & \pm & 12.65 & 0.1338 \\
\hline Disabled (no grade, grade 4-7) & 161 & 4.5 & 14.41 & \pm & 11.87 & & 8 & 2.7 & 11.82 & \pm & 9.72 & \\
\hline Disabled (grade 1-3) & 290 & 8.1 & 10.56 & \pm & 10.31 & & 21 & 7.0 & 19.74 & \pm & 12.13 & \\
\hline Chronic diseases & & & & & & & & & & & & \\
\hline No & 1,868 & 52.4 & 6.49 & \pm & 7.61 & $<.0001$ & 86 & 28.9 & 11.63 & \pm & 11.93 & 0.0932 \\
\hline Yes & 1,700 & 47.6 & 9.70 & \pm & 10.16 & & 212 & 71.1 & 17.44 & \pm & 12.47 & \\
\hline
\end{tabular}

*NBLGS the National Basic Livelihood Guarantee System 
Table 2 Generalized estimating equation (GEE) analysis of factors associated with CESD-11

\begin{tabular}{|c|c|c|c|c|c|c|}
\hline \multirow[t]{3}{*}{ Variables } & \multicolumn{6}{|c|}{ CESD-11 } \\
\hline & \multicolumn{3}{|l|}{ Male } & \multicolumn{3}{|c|}{ Female } \\
\hline & ß & S.E & $P$ value & ß & S.E & $P$ value \\
\hline \multicolumn{7}{|l|}{ Change in material hardship } \\
\hline $\mathrm{No} \rightarrow \mathrm{No}$ & Ref & & & Ref & & \\
\hline $\mathrm{No} \rightarrow \mathrm{Yes}$ & 2.28 & 0.20 & $<.0001$ & 3.41 & 0.26 & $<.0001$ \\
\hline $\mathrm{Yes} \rightarrow \mathrm{No}$ & 0.45 & 0.16 & 0.0045 & 0.84 & 0.21 & $<.0001$ \\
\hline Yes $\rightarrow$ Yes & 2.82 & 0.24 & $<.0001$ & 3.98 & 0.30 & $<.0001$ \\
\hline \multicolumn{7}{|l|}{ Prior year depression status } \\
\hline No & Ref & & & Ref & & \\
\hline Yes & 3.14 & 0.19 & $<.0001$ & 2.93 & 0.18 & $<.0001$ \\
\hline \multicolumn{7}{|l|}{ Age (years) } \\
\hline $19-29$ & Ref & & & Ref & & \\
\hline $30-39$ & 0.84 & 0.19 & $<.0001$ & 0.19 & 0.22 & 0.3794 \\
\hline $40-49$ & 1.30 & 0.20 & $<.0001$ & 0.02 & 0.24 & 0.9372 \\
\hline $50-59$ & 1.95 & 0.24 & $<.0001$ & 0.76 & 0.28 & 0.0068 \\
\hline $60-69$ & 2.30 & 0.26 & $<.0001$ & 1.09 & 0.32 & 0.0007 \\
\hline$\geq 70$ & 3.52 & 0.27 & $<.0001$ & 2.90 & 0.33 & $<.0001$ \\
\hline \multicolumn{7}{|l|}{ Region } \\
\hline Metropolitan & Ref & & & Ref & & \\
\hline Rural & -0.17 & 0.11 & 0.1190 & -0.04 & 0.13 & 0.7340 \\
\hline \multicolumn{7}{|l|}{ Education level } \\
\hline Middle school or under & 0.72 & 0.19 & 0.0002 & 1.44 & 0.25 & $<.0001$ \\
\hline High school & 0.10 & 0.13 & 0.4331 & 0.33 & 0.17 & 0.0496 \\
\hline College or above & Ref & & & Ref & & \\
\hline \multicolumn{7}{|l|}{ Marital status } \\
\hline Living w spouse & Ref & & & Ref & & \\
\hline Living w/o spouse & 2.15 & 0.15 & $<.0001$ & 1.36 & 0.16 & $<.0001$ \\
\hline \multicolumn{7}{|l|}{ Income level } \\
\hline Low & 1.98 & 0.15 & $<.0001$ & 2.05 & 0.18 & $<.0001$ \\
\hline Lower middle & 1.00 & 0.11 & $<.0001$ & 1.13 & 0.14 & $<.0001$ \\
\hline Upper middle & 0.56 & 0.09 & $<.0001$ & 0.53 & 0.12 & $<.0001$ \\
\hline High & Ref & & & Ref & & \\
\hline \multicolumn{7}{|l|}{ NBLGS* status } \\
\hline Non-beneficiary & Ref & & & Ref & & \\
\hline Beneficiary & 2.05 & 0.30 & $<.0001$ & 1.93 & 0.30 & $<.0001$ \\
\hline \multicolumn{7}{|l|}{ Current smoking status } \\
\hline Non-smoker & Ref & & & Ref & & \\
\hline Current smoker & 0.69 & 0.10 & $<.0001$ & 1.72 & 0.40 & $<.0001$ \\
\hline \multicolumn{7}{|l|}{ Alcohol consumption } \\
\hline Non-drinkers & Ref & & & Ref & & \\
\hline Once a week or less & -0.59 & 0.11 & $<.0001$ & -0.11 & 0.12 & 0.3700 \\
\hline 2-3 times a week & -0.70 & 0.13 & $<.0001$ & 0.09 & 0.23 & 0.6883 \\
\hline More than 4 times a week & -0.26 & 0.16 & 0.0920 & 0.59 & 0.43 & 0.1741 \\
\hline \multicolumn{7}{|l|}{ Disability status } \\
\hline Not disabled & Ref & & & Ref & & \\
\hline Disabled (no grade, grade 4-7) & 0.96 & 0.22 & $<.0001$ & 1.09 & 0.33 & 0.0011 \\
\hline Disabled (grade 1-3) & 2.62 & 0.40 & $<.0001$ & 2.05 & 0.55 & 0.0002 \\
\hline \multicolumn{7}{|l|}{ Chronic diseases } \\
\hline No & Ref & & & Ref & & \\
\hline
\end{tabular}


Table 2 (continued)

\begin{tabular}{|c|c|c|c|c|c|c|}
\hline \multirow[t]{3}{*}{ Variables } & \multicolumn{6}{|c|}{ CESD-11 } \\
\hline & \multicolumn{3}{|l|}{ Male } & \multicolumn{3}{|c|}{ Female } \\
\hline & B & S.E & $P$ value & B & S.E & $P$ value \\
\hline Yes & 1.02 & 0.09 & $<.0001$ & 1.23 & 0.13 & $<.0001$ \\
\hline \multicolumn{7}{|l|}{ Year } \\
\hline 2009 & Ref & & & Ref & & \\
\hline 2010 & -1.10 & 0.17 & $<.0001$ & 0.95 & 0.84 & 0.2608 \\
\hline 2011 & -1.52 & 0.16 & $<.0001$ & -0.01 & 0.82 & 0.9913 \\
\hline 2012 & -2.32 & 0.16 & $<.0001$ & -2.45 & 0.73 & 0.0008 \\
\hline 2013 & -1.97 & 0.16 & $<.0001$ & -2.57 & 0.69 & 0.0002 \\
\hline 2014 & -1.09 & 0.16 & $<.0001$ & -1.90 & 0.69 & 0.0060 \\
\hline 2015 & -2.43 & 0.16 & $<.0001$ & -2.96 & 0.70 & $<.0001$ \\
\hline 2016 & -2.34 & 0.17 & $<.0001$ & -2.99 & 0.69 & $<.0001$ \\
\hline 2017 & -2.28 & 0.17 & $<.0001$ & -3.17 & 0.69 & $<.0001$ \\
\hline
\end{tabular}

*NBLGS The National Basic Livelihood Guarantee System

groups. Among male participants who were depressed in the prior year and had a low income level, both the $\mathrm{No} \rightarrow$ Yes and the Yes $\rightarrow$ Yes groups had higher risk of depression status even compared to the result from Table 2. A*mong female participants who had a low income level, were disabled, had a low educational level, and were suffering from chronic diseases, higher risk of depression status in the No $\rightarrow$ Yes and the Yes $\rightarrow$ Yes groups were similarly observed.

Table 4 shows the results of effects of $\mathrm{MH}$ on depression based on the dimensions of $\mathrm{MH}$. In the $\mathrm{MH}$ subcategories associated with food, paying utility bills, and financial hardship, male participants who continually experienced $\mathrm{MH}$ showed the highest depression scores (food hardship: $\beta=3.29$; paying utility bills hardship: $\beta=1.53$; financial hardship: $\beta=0.87$ ), while the No $\rightarrow$ Yes group showed the second highest scores (food hardship: $\beta=2.46$; paying utility bills hardship: $\beta=1.02$; financial hardship: $\beta=0.68$ ). For housing hardship, the $\mathrm{No} \rightarrow$ Yes group showed higher depression scores $(\beta=2.02)$ than the Yes $\rightarrow$ Yes group. In terms of food, and medical care hardship, female participants who continually experienced $\mathrm{MH}$ showed the highest depression scores (food hardship: $\beta=4.05$; medical care hardship: $\beta=3.63$ ), followed by the No $\rightarrow$ Yes group (food hardship: $\beta=3.70$; medical care hardship: $\beta=2.66)$. As for housing and financial hardship, the No $\rightarrow$ Yes group showed a higher depression score (housing hardship: $\beta=3.36$, financial hardship: $\beta=1.09$ ) than the Yes $\rightarrow$ Yes group.

\section{Discussion}

In this study, we examined the effect of $\mathrm{MH}$ on depression. The main finding of the present study suggests that changes in experiencing $\mathrm{MH}$ and constantly experiencing
$\mathrm{MH}$ are related to an increased risk of depression. A change from Yes to No was found to be slightly related to an increased risk of depression. Both a change from No to Yes and a change from Yes to No were strongly related to an increased risk of depression. Moreover, the $\mathrm{MH} \mathrm{No} \rightarrow$ Yes and Yes $\rightarrow$ Yes groups who were NBLGS beneficiaries, disabled, experienced depression in the prior year, had lower income levels, and chronic diseases were at greater risk of depression compared with those who did not belong to any of these demographic groups, regardless of their gender. Furthermore, food hardship, one of the dimensions of $\mathrm{MH}$, was found to be strongly associated with depression, more than any other factor, while education hardship was not statistically associated with depression. Females were found to be more at risk of depression than males, with females showing higher depression scores than males at the first time point.

In line with this finding, previous studies have shown that $\mathrm{MH}$ is an important risk factor for mental health in low-income households [14, 23]. Ross and Huber [24] pointed out that daily struggles to meet basic needs due to limited financial resources may result in experiencing exhaustion, despair, and depression. The previous study used the first two years (2006 and 2007) of KOWEPS to identify the effect of experiencing $\mathrm{MH}$ in 2006 on depression status in 2007 using lagged dependent variable model [25]. The result is consistent with our findings showing that $\mathrm{MH}$, especially food hardship, is associated with depression. However, our study identified the transition of experiencing $\mathrm{MH}$ over two consecutive years repeatedly using a panel data after adjusting demographic, socioeconomic, and health related factors.

Food hardship had the most salient influence on depression in our study. This subgroup analysis result 
Table 3 Subgroup analysis of CESD-11 stratified by covariates

\begin{tabular}{|c|c|c|c|c|c|c|c|c|c|c|}
\hline \multirow[t]{3}{*}{ Variables } & \multicolumn{10}{|l|}{ CESD-11 } \\
\hline & \multirow{2}{*}{$\begin{array}{l}\text { No } \rightarrow \text { No } \\
B^{*}\end{array}$} & \multicolumn{3}{|c|}{ No $\rightarrow$ Yes } & \multicolumn{3}{|c|}{ Yes $\rightarrow$ Yes } & \multicolumn{3}{|c|}{ Yes $\rightarrow$ Yes } \\
\hline & & $\beta^{*}$ & S.E & $P$ value & $B^{*}$ & S.E & $P$ value & $B^{*}$ & S.E & $P$ value \\
\hline \multicolumn{11}{|l|}{ Male } \\
\hline \multicolumn{11}{|l|}{ Prior year depression status } \\
\hline No & Ref & 2.13 & 0.21 & $<.0001$ & 0.49 & 0.16 & 0.0020 & 2.51 & 0.25 & $<.0001$ \\
\hline Yes & Ref & 2.83 & 0.66 & $<.0001$ & -0.03 & 0.52 & 0.9490 & 3.26 & 0.56 & $<.0001$ \\
\hline \multicolumn{11}{|l|}{ Income level } \\
\hline Low & Ref & 3.14 & 0.36 & $<.0001$ & 0.62 & 0.33 & 0.0621 & 3.43 & 0.40 & $<.0001$ \\
\hline Lower middle & Ref & 2.44 & 0.39 & $<.0001$ & 0.33 & 0.30 & 0.2609 & 2.83 & 0.40 & $<.0001$ \\
\hline Upper middle & Ref & 1.57 & 0.39 & $<.0001$ & 0.35 & 0.30 & 0.2486 & 2.06 & 0.42 & $<.0001$ \\
\hline High & Ref & 0.77 & 0.43 & 0.0751 & 0.67 & 0.33 & 0.0426 & 2.56 & 0.62 & $<.0001$ \\
\hline \multicolumn{11}{|l|}{ NBLGS** status } \\
\hline Non-beneficiary & Ref & 2.26 & 0.22 & $<.0001$ & 0.54 & 0.16 & 0.0010 & 2.61 & 0.25 & $<.0001$ \\
\hline Beneficiary & Ref & 2.38 & 0.62 & 0.0001 & 0.16 & 0.56 & 0.7744 & 3.06 & 0.59 & $<.0001$ \\
\hline \multicolumn{11}{|l|}{ Disability status } \\
\hline Not disabled & Ref & 2.18 & 0.22 & $<.0001$ & 0.36 & 0.17 & 0.0348 & 2.80 & 0.25 & $<.0001$ \\
\hline Disabled (no grade, grade 4-7) & Ref & 2.68 & 0.74 & 0.0003 & 0.79 & 0.55 & 0.1495 & 2.66 & 0.77 & 0.0005 \\
\hline Disabled (grade 1-3) & Ref & 2.40 & 0.85 & 0.0050 & 0.76 & 0.77 & 0.3288 & 3.25 & 0.96 & 0.0007 \\
\hline \multicolumn{11}{|l|}{ Chronic diseases } \\
\hline No & Ref & 1.65 & 0.26 & $<.0001$ & 0.42 & 0.20 & 0.0387 & 2.38 & 0.29 & $<.0001$ \\
\hline Yes & Ref & 2.80 & 0.30 & $<.0001$ & 0.48 & 0.25 & 0.0563 & 3.22 & 0.35 & $<.0001$ \\
\hline \multicolumn{11}{|l|}{ Female } \\
\hline \multicolumn{11}{|l|}{ Prior year depression status } \\
\hline No & Ref & 2.74 & 0.27 & $<.0001$ & 1.10 & 0.23 & $<.0001$ & 2.97 & 0.32 & $<.0001$ \\
\hline Yes & Ref & 5.33 & 0.59 & $<.0001$ & 0.34 & 0.47 & 0.4687 & 5.18 & 0.56 & $<.0001$ \\
\hline \multicolumn{11}{|l|}{ Income level } \\
\hline Low & Ref & 4.39 & 0.40 & $<.0001$ & 0.93 & 0.35 & 0.0081 & 4.92 & 0.44 & $<.0001$ \\
\hline Lower middle & Ref & 3.08 & 0.49 & $<.0001$ & 0.74 & 0.40 & 0.0662 & 3.60 & 0.53 & $<.0001$ \\
\hline Upper middle & Ref & 2.25 & 0.57 & $<.0001$ & 1.45 & 0.49 & 0.0028 & 1.79 & 0.65 & 0.0061 \\
\hline High & Ref & 1.89 & 0.67 & 0.0045 & 0.51 & 0.46 & 0.2654 & 4.01 & 0.92 & $<.0001$ \\
\hline \multicolumn{11}{|l|}{ NBLGS** status } \\
\hline Non-beneficiary & Ref & 3.28 & 0.28 & $<.0001$ & 0.86 & 0.23 & 0.0001 & 4.17 & 0.36 & $<.0001$ \\
\hline Beneficiary & Ref & 3.62 & 0.60 & $<.0001$ & 0.83 & 0.53 & 0.1169 & 3.54 & 0.56 & $<.0001$ \\
\hline \multicolumn{11}{|l|}{ Disability status } \\
\hline Not disabled & Ref & 3.38 & 0.27 & $<.0001$ & 0.79 & 0.22 & 0.0003 & 3.96 & 0.31 & $<.0001$ \\
\hline Disabled (no grade, grade $4-7$ ) & Ref & 4.15 & 1.00 & $<.0001$ & 0.97 & 0.88 & 0.2698 & 4.78 & 1.26 & 0.0001 \\
\hline Disabled (grade 1-3) & Ref & 3.27 & 1.27 & 0.0097 & 2.40 & 1.13 & 0.0338 & 3.79 & 1.32 & 0.0041 \\
\hline \multicolumn{11}{|l|}{ Chronic diseases } \\
\hline No & Ref & 2.00 & 0.38 & $<.0001$ & 0.48 & 0.30 & 0.1078 & 2.37 & 0.43 & $<.0001$ \\
\hline Yes & Ref & 4.17 & 0.33 & $<.0001$ & 1.09 & 0.28 & $<.0001$ & 4.89 & 0.38 & $<.0001$ \\
\hline
\end{tabular}

${ }^{*}$ All other covariates were adjusted

**NBLGS The National Basic Livelihood Guarantee System

was similar to the results demonstrated by previous studies [26, 27]. The lack of significant association between depression scores and education hardship and problems with paying utility bills could be explained by the following. First, the sample size for those dimensions may have been too small to determine the nature of the relationship between these factors and depressive symptoms. Second, especially for education hardship, there is generally freedom from financial burdens associated with tuition. In Korea, tuition for public elementary and middle schools are fully supported by the state. Even for higher education, numerous companies subsidise or 
Table 4 GEE analysis of each material hardship dimension

\begin{tabular}{|c|c|c|c|c|c|c|c|c|c|c|}
\hline \multirow[t]{3}{*}{ Material hardship dimensions } & \multicolumn{10}{|l|}{ CESD-11 } \\
\hline & \multirow{2}{*}{$\begin{array}{l}\mathrm{No} \rightarrow \mathrm{No} \\
\beta^{*}\end{array}$} & \multicolumn{3}{|c|}{ No $\rightarrow$ Yes } & \multicolumn{3}{|c|}{ Yes $\rightarrow$ No } & \multicolumn{3}{|c|}{ Yes $\rightarrow$ Yes } \\
\hline & & $\beta^{*}$ & S.E & $P$ value & $\beta^{*}$ & S.E & $P$ value & $\beta^{*}$ & S.E & $P$ value \\
\hline \multicolumn{11}{|l|}{ Male } \\
\hline Food hardship & Ref & 2.46 & 0.26 & $<.0001$ & 0.67 & 0.21 & 0.0016 & 3.29 & 0.43 & $<.0001$ \\
\hline Housing hardship & Ref & 2.02 & 0.57 & 0.0004 & 0.13 & 0.45 & 0.7678 & 1.54 & 0.75 & 0.0408 \\
\hline Paying utilization bills hardship & Ref & 1.02 & 0.36 & 0.0047 & 0.37 & 0.25 & 0.1380 & 1.53 & 0.58 & 0.0083 \\
\hline Financial hardship & Ref & 0.68 & 0.35 & 0.0478 & -0.10 & 0.28 & 0.7258 & 0.87 & 0.32 & 0.0065 \\
\hline Education hardship & Ref & 0.01 & 0.72 & 0.9859 & 1.35 & 0.78 & 0.0817 & 0.52 & 1.61 & 0.7468 \\
\hline Medical care hardship & Ref & 1.54 & 0.48 & 0.0012 & 0.28 & 0.38 & 0.4718 & -0.44 & 0.84 & 0.6005 \\
\hline \multicolumn{11}{|l|}{ Female } \\
\hline Food hardship & Ref & 3.70 & 0.31 & $<.0001$ & 1.08 & 0.27 & $<.0001$ & 4.05 & 0.44 & $<.0001$ \\
\hline Housing hardship & Ref & 3.36 & 0.68 & $<.0001$ & 0.42 & 0.60 & 0.4873 & 3.30 & 1.15 & 0.0040 \\
\hline Paying utilization bills hardship & Ref & 0.90 & 0.47 & 0.0570 & -0.19 & 0.39 & 0.6189 & 1.10 & 0.76 & 0.1510 \\
\hline Financial hardship & Ref & 1.09 & 0.51 & 0.0319 & 0.25 & 0.36 & 0.4831 & 0.90 & 0.45 & 0.0430 \\
\hline Education hardship & Ref & 0.49 & 1.08 & 0.6481 & -1.15 & 0.96 & 0.2311 & -1.58 & 1.87 & 0.3978 \\
\hline Medical care hardship & Ref & 2.66 & 0.64 & $<.0001$ & 0.12 & 0.52 & 0.8239 & 3.63 & 1.46 & 0.0133 \\
\hline
\end{tabular}

* All covariates were adjusted

provide scholarships for their employees' children as a part of company internal welfare systems.

The effect size of $\mathrm{MH}$ on depression differed by gender. In both genders, food hardship was the most influential dimension of $\mathrm{MH}$. This might be related to the gender-role in Korea. Although the participation in the workforce by women has been rapidly growing in Korea, the labor force participation rate of women (52.8\%) was distinctly lower than that of men (72.6\%) in 2020 [28]. Korean traditions remain strong in families despite many socioeconomic changes. Men regarded domestic work as shameful, defaming masculinity; therefore, doing household chores was treated as a mandatory for women [29]. Because of this "traditional gender-role," female participants might be much more influenced by $\mathrm{MH}$, especially food hardship, compared to male participants. It might be associated with the different effect size of $\mathrm{MH}$ by gender.

One interesting result was that participants who experienced $\mathrm{MH}$ in the prior year but no longer experienced $\mathrm{MH}$ in the following year still showed higher depression scores than those who had never experienced MH. Stigma and uncertainty about one's future MH status might be a possible explanation. Mickelson suggested that internalised stigma (individual's personal negative feelings about their poverty) and experienced stigma (individual's perception of being treated as stigmatised by behaviours and feelings of others) are related to depression as mediators. Furthermore, internalised stigma and depression were partially mediated by self-esteem and fear of rejection, while experienced stigma was related to depression through fear of rejection only [30]. Our results might reflect the process of suffering those stigmas and recovering from them. The uncertainty about one's future MH status, which is another possible explanation, may be related to stress. First, pre-experience of social exclusion was found to lead to a blunted cortisol response to stress, which might contribute to higher vulnerability to health disturbances [31]. Second, persistent uncertainty about the future might lead to development of a persistent cerebral energy crisis, contributing to systemic and brain malfunction. When people feel uncertain, they anticipate that outcomes will turn out to be unexpected and feel unable to avoid surprise, and all their cognitive systems strive to reduce it by using cerebral energy. Moreover, the 'selfish brain' demands extra energy from the body in times of uncertainty. So, if the brain cannot reduce uncertainty, a persistent cerebral energy crisis may develop into systemic and brain malfunction [32].

The small size of female participants might be related to the self-reported smoking status in this data. In Korea, other self-reported data had inaccurate self-reporting of smoking habits in females [33]. In our study, most female subjects were excluded from the total sample due to the missing value in smoking status. To identify the effect $\mathrm{MH}$ change on depression adjusting smoking status in females, further study using data obtained from other smoking detection methods, e.g. urine cotinine examination should be considered.

This study has several limitations. First, because of data limitation, we could not use objective measurement tools 
for material deprivation such as the Townsend index, which is a widely used and valid measure of material deprivation [34, 35]. However, we used $\mathrm{MH}$ measures based on a pervious study [14]. Second, because the KoWePS data largely represent low-income households, the findings are somewhat limited when it comes to generalising to the overall population, particularly high-income households. Third, although we used several control variables to reduce the omitted variable bias, there still might be some unobserved confounding factors that prevent confirming a causal relationship with certainty.

Despite the limitations, some strengths are notable. First, this study was based on the second-largest open access panel survey data in South Korea. Many experts have verified its high validity and national representativeness. As the KoWePS largely represents low-income households and randomly sampled participants nationwide, our findings could be generalised to the South Korean low-income population. Second, to expressly incorporate the financial struggles that low-income households face, we used $\mathrm{MH}$ as an index of economic hardship as a substitute for a conventional one-dimensional income-based measure. Most previous studies in Korea have focussed on the association between a type of $\mathrm{MH}$, mainly food insecurity and mental health [36, 37]. However, our study looked at the broader effects of economic difficulties on depression using six dimensions of $\mathrm{MH}$, including two uniquely Korean aspects. Thus, this study contributes to the literature by using $\mathrm{MH}$ as a measure of multidimensional financial struggles among low-income households in South Korea.

\section{Conclusions}

In conclusion, this study identified the effects of $\mathrm{MH}$ on depression by gender, and the results were significant. We found that people who had experienced $\mathrm{MH}$ at least once had a higher risk for depression. Especially, people who continually experienced $\mathrm{MH}$ were at the highest risk for depression. Finally, it was found that food hardship was strongly associated with depression. Based on this study, we should consider guaranteeing food security for low-income populations to reduce the incidence of depression. In addition, community and policy makers should consider MH in their approaches to identifying populations at high risk of depression and people encountering financial struggles.

\footnotetext{
Abbreviations

OECD: Organisation for Economic Cooperation and Development; CESD-11: Center for Epidemiologic Studies Depression Scale; MH: Material hardship; KoWePS: Korea Welfare Panel Study; NBLGS: National Basic Livelihood Guarantee System; ANOVA: Analysis of variance; GEE: Generalised estimating equation.
}

\section{Acknowledgements}

We are grateful to the Korea Institute for Health and Social Affairs and Seoul National University that conducted KoWePS which is the primary source of our study.

\section{Authors' contributions}

$\mathrm{KSH}$ and JSI designed the study. KSH wrote the main manuscript text. All authors prepared the tables and reviewed the manuscript. The author(s) read and approved the final manuscript.

\section{Funding}

This research was supported by a grant of the Korea Health Technology R\&D Project through the Korea Health Industry Development Institute (KHIDI), funded by the Ministry of Health \& Welfare, Republic of Korea (grant number: HI20C1130)

\section{Availability of data and materials}

The dataset is available on the Korea Welfare Panel Study (KoWePS) website: https://www.koweps.re.kr:442/main.do.

\section{Declarations}

\section{Ethics approval and consent to participate}

This study was approved by the Institutional Review Board of Korea Institute for Health and Social Affairs (No. 2021-002). This data is publicly accessible and written informed consent is obtained from all the participants before participating in the survey. Respondents' information was completely anonymized for use for research purposes and unidentified prior to analysis. The authors assert that all procedures contributing to this work comply with the ethical standards of the relevant national and institutional committees on human experimentation and with the Helsinki Declaration of 1975, as revised in 2000.

\section{Consent for publication}

Not applicable.

\section{Competing interests}

The authors declare that they have no competing interests.

\section{Author details}

1 Department of Public Health, Graduate School, Yonsei University, Seoul, Republic of Korea. ${ }^{2}$ Institute of Health Services Research, Yonsei University, Seoul, Republic of Korea. ${ }^{3}$ Department of Preventive Medicine, Yonsei University College of Medicine, 50 Yonsei-ro, Seodaemun-gu, Seoul 03722, Republic of Korea.

Received: 23 February 2021 Accepted: 11 August 2021

Published online: 07 September 2021

\section{References}

1. Ku I, et al. The role of family behaviors in determining income distribution: the case of South Korea. Demography. 2018;55(3):877-99.

2. Jung HS. Changes and causes of income inequality in OECD countries. Health Welfare Policy Forum. 2011;179:106-16.

3. Lee $\mathbf{W}$. The poverty reduction effect of the national basic livelihood security system. Health Welfare Forum. 2021;2(4):27-40.

4. Organisation for Economic Cooperation and Development (OECD), Social spending (indicator). Paris: OECD; 2020.

5. Ouellette T, Burstein N, Long D, Beecroft E. Measures of material hardship: Final report. Washington, DC: US Department of Health and Human Services, Office of the Assistant Secretary for Planning and Evaluation; 2004.

6. Kim J, Lee R, Shim J. Material hardship and self-rated health among low-income households in South Korea. Asia Pac J Soc Work. 2018;28(4):279-94.

7. Sullivan JX, Turner L, Danziger S. The relationship between income and material hardship. J Policy Anal Manage. 2008;27(1):63-81.

8. Sen A. Issues in the Measurement of Poverty. Scand J Econ. 1979;81(2):285-307.

9. Townsend P. The Meaning of Poverty. Br J Sociol. 1962;13(3):210-27. 
10. Townsend P. Poverty in the United Kingdom: a survey of household resources and standards of living. California: University of California Press; 1979

11. Beverly SG. Measures of material hardship. J Poverty. 2001;5(1):23-41.

12. Beverly SG. Material hardship in the United States: evidence from the survey of income and program participation. Soc Work Res. 2001;25(3):143-51.

13. World Health Organization. Investing in mental health. Geneva: World Health Organization; 2003

14. Heflin CM, Iceland J. Poverty, material hardship, and depression. Soc Sci Q. 2009;90(5):1051-71.

15. Kim W, et al. The effect of childhood and current economic status on depressive symptoms in South Korean individuals: a longitudinal study. Int J Equity Health. 2016;15(1):111.

16. Song A, Kim W. The association between relative income and depressive symptoms in adults: findings from a nationwide survey in Korea. J Affect Disord. 2020;263:236-40.

17. Ju YJ, et al. Low socioeconomic status and suicidal ideation among elderly individuals. Int Psychogeriatr. 2016;28(12):2055-66.

18. Kim $\mathrm{J}$, et al. Effect of socioeconomic status on the linkage between suicidal ideation and suicide attempts. Suicide Life-Threat Behav. 2016;46(5):588-97.

19. Kohout FJ, et al. Two shorter forms of the CES-D depression symptoms index. J Aging Health. 1993;5(2):179-93.

20. Cho MJ, Kim KH. Diagnostic validity of the CES-D (Korean version) in the assessment of DSM-III-R major depression. J Korean Neuropsychiatr Assoc. 1993;32(3):381-99.

21. Weissman MM, et al. Assessing depressive symptoms in five psychiatric populations: a validation study. Am J Epidemiol. 1977;106(3):203-14

22. Ballinger GA. Using generalized estimating equations for longitudinal data analysis. Organ Res Methods. 2004;7(2):127-50.

23. Butterworth $P$, Rodgers $B$, Windsor TD. Financial hardship, socio-economic position and depression: results from the PATH Through Life Survey. Soc Sci Med. 2009;69(2):229-37.

24. Ross CE, Huber J. Hardship and depression. J Health Soc Behav. 1985;26(4):312-27. https://www.jstor.org/stable/2136655.

25. Kim J, Shim J, Lee R. Material hardship and depression among lowincome households in South Korea: Differences by household type. Int Soc Welfare. 2016;25(2):187-98.
26. Johnson CM, Sharkey JR, Dean WR. Indicators of material hardship and depressive symptoms among homebound older adults living in North Carolina. J Nutr Gerontol Geriatr. 2011;30(2):154-68.

27. Alley DE, et al. Material resources and population health: disadvantages in health care, housing, and food among adults over 50 years of age. Am J Public Health. 2009;99(S3):S693-701.

28. Statistics Korea, Economically active population survey. Daejeon: Statisitcs Korea, Social Statistics Bureau, Employment Statistics Division; 2020.

29. Sung S. Women Reconciling Paid and Unpaid Work in a Confucian Welfare State: The Case of South Korea. Soc Policy Admin. 2003;37(4):342-60.

30. Mickelson KD, Williams SL. Perceived stigma of poverty and depression: examination of interpersonal and intrapersonal mediators. J Soc Clin Psychol. 2008;27(9):903-30

31. Weik $U$, et al. Pre-experience of social exclusion suppresses cortisol response to psychosocial stress in women but not in men. Horm Behav. 2010;58(5):891-7.

32. Peters A, McEwen BS, Friston K. Uncertainty and stress: Why it causes diseases and how it is mastered by the brain. Prog Neurobiol. 2017;156:164-88.

33. Kang $\mathrm{HG}$, et al. Biochemically-verified smoking rate trends and factors associated with inaccurate self-reporting of smoking habits in Korean women. Asian Pac J Cancer Prev. 2013;14(11):6807-12.

34. Gordon D. Area-based deprivation measures: a UK perspective. Neighborhoods and health, ed. K. I and B. LF. New York: Oxford University Press; 2003.

35. Townsend P, Phillimore $P$, Beattie $A$. Health and deprivation: inequality and the North. UK: Routledge; 1988.

36. Lee YS, Kim TH. Household food insecurity and breakfast skipping: Their association with depressive symptoms. Psychiatry Res. 2019;271:83-8.

37. Leung CW, et al. Household food insecurity is positively associated with depression among low-income supplemental nutrition assistance program participants and income-eligible nonparticipants. J Nutr. 2015;145(3):622-7.

\section{Publisher's Note}

Springer Nature remains neutral with regard to jurisdictional claims in published maps and institutional affiliations.
Ready to submit your research? Choose BMC and benefit from:

- fast, convenient online submission

- thorough peer review by experienced researchers in your field

- rapid publication on acceptance

- support for research data, including large and complex data types

- gold Open Access which fosters wider collaboration and increased citations

- maximum visibility for your research: over 100M website views per year

At $\mathrm{BMC}$, research is always in progress.

Learn more biomedcentral.com/submissions 